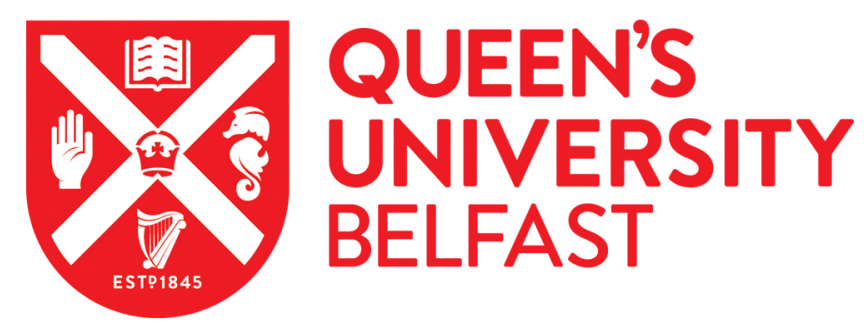

\title{
Modified Thomson spectrometer design for high energy, multi-species ion sources
}

Gwynne, D., Kar, S., Doria, D., Ahmed, H., Cerchez, M., Fernandez, J., Gray, R. J., Green, J. S., Hanton, F., MacLellan, D. A., McKenna, P., Najmudin, Z., Neely, D., Ruiz, J. A., Schiavi, A., Streeter, M., Swantusch, M., Willi, O., Zepf, M., \& Borghesi, M. (2014). Modified Thomson spectrometer design for high energy, multi-species ion sources. Review of Scientific Instruments, 85(3), [033304]. https://doi.org/10.1063/1.4866021

Published in:

Review of Scientific Instruments

Document Version:

Publisher's PDF, also known as Version of record

Queen's University Belfast - Research Portal:

Link to publication record in Queen's University Belfast Research Portal

\section{Publisher rights}

Copyright 2014 American Institute of Physics. This article may be downloaded for personal use only. Any other use requires prior permission of the author and the American Institute of Physics.

The following article appeared in Rev. Sci. Instrum. 85, 033304 (2014) and may be found at http://scitation.aip.org/content/aip/journal/rsi/85/3/10.1063/1.4866021

\section{General rights}

Copyright for the publications made accessible via the Queen's University Belfast Research Portal is retained by the author(s) and / or other copyright owners and it is a condition of accessing these publications that users recognise and abide by the legal requirements associated with these rights.

\section{Take down policy}

The Research Portal is Queen's institutional repository that provides access to Queen's research output. Every effort has been made to ensure that content in the Research Portal does not infringe any person's rights, or applicable UK laws. If you discover content in the Research Portal that you believe breaches copyright or violates any law, please contact openaccess@qub.ac.uk. 


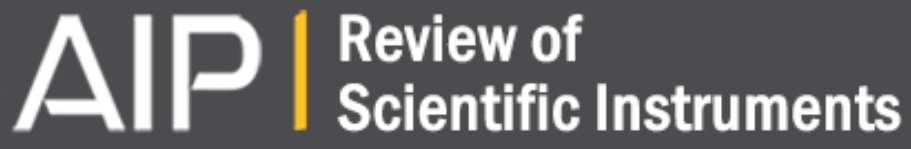

\section{Modified Thomson spectrometer design for high energy, multi-species ion sources}

D. Gwynne, S. Kar, D. Doria, H. Ahmed, M. Cerchez, J. Fernandez, R. J. Gray, J. S. Green, F. Hanton, D. A. MacLellan, P. McKenna, Z. Najmudin, D. Neely, J. A. Ruiz, A. Schiavi, M. Streeter, M. Swantusch, O. Willi, M. Zepf, and M. Borghesi

Citation: Review of Scientific Instruments 85, 033304 (2014); doi: 10.1063/1.4866021

View online: http://dx.doi.org/10.1063/1.4866021

View Table of Contents: http://scitation.aip.org/content/aip/journal/rsi/85/3?ver=pdfcov

Published by the AIP Publishing

\section{Articles you may be interested in}

The study towards high intensity high charge state laser ion sourcesa)

Rev. Sci. Instrum. 85, 02B910 (2014); 10.1063/1.4833921

Review of laser ion sources developments in Prague and production of q over 50+ ions at Prague Asterix Laser System (invited)

Rev. Sci. Instrum. 75, 1546 (2004); 10.1063/1.1695646

Production of He-like light and medium mass ions in laser ion source

Rev. Sci. Instrum. 71, 1409 (2000); 10.1063/1.1150471

Development of a high-current laser ion source for induction accelerators

Rev. Sci. Instrum. 71, 1216 (2000); 10.1063/1.1150437

Negative ion sources for high energy accelerators (invited)

Rev. Sci. Instrum. 71, 1069 (2000); 10.1063/1.1150388

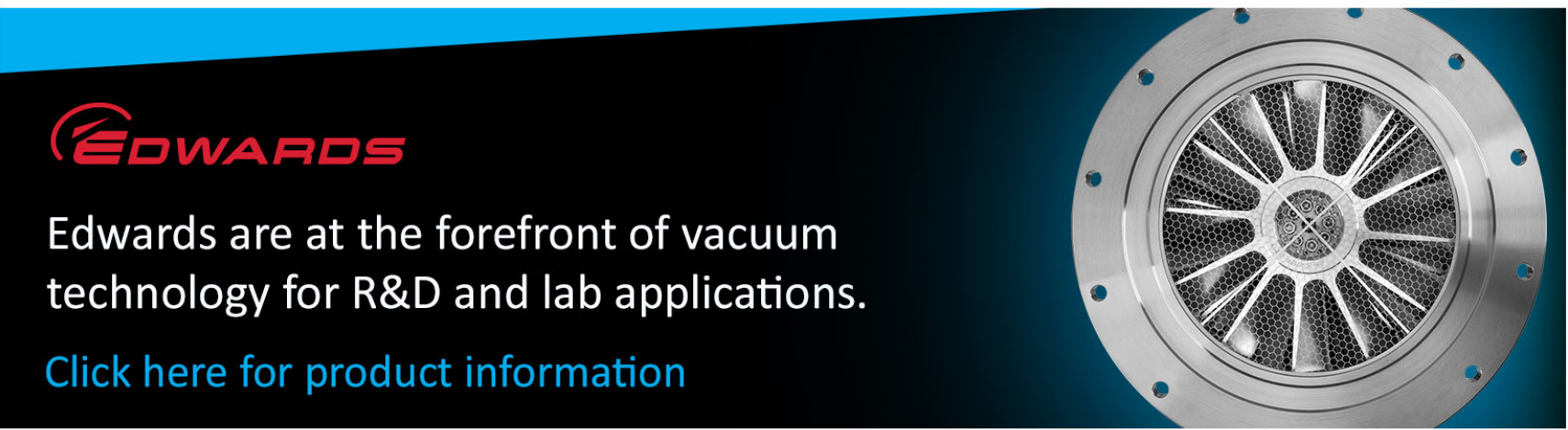




\title{
Modified Thomson spectrometer design for high energy, multi-species ion sources
}

\author{
D. Gwynne, ${ }^{1}$ S. Kar, ${ }^{1, a)}$ D. Doria, ${ }^{1}$ H. Ahmed, ${ }^{1}$ M. Cerchez, ${ }^{2}$ J. Fernandez, ${ }^{3,4}$ R. J. Gray, ${ }^{5}$ \\ J. S. Green, ${ }^{4}$ F. Hanton, ${ }^{1}$ D. A. MacLellan, ${ }^{5}$ P. McKenna, ${ }^{5}$ Z. Najmudin, ${ }^{6}$ D. Neely, ${ }^{4}$ J. A. \\ Ruiz, ${ }^{3}$ A. Schiavi, ${ }^{7}$ M. Streeter, ${ }^{6}$ M. Swantusch, ${ }^{2}$ O. Willi, ${ }^{2}$ M. Zepf, ${ }^{1,8}$ and M. Borghesi ${ }^{1,9}$ \\ ${ }^{1}$ Centre for Plasma Physics, School of Mathematics and Physics, Queen's University Belfast, \\ Belfast BT7 1NN, United Kingdom \\ ${ }^{2}$ Institut für Laser-und Plasmaphysik, Heinrich-Heine-Universität, Düsseldorf, Germany \\ ${ }^{3}$ Instituto de Fusión Nuclear UPM, Jose Gutierrez, Abascal 2, E28006 Madrid, Spain \\ ${ }^{4}$ Central Laser Facility, Rutherford Appleton Laboratory, Didcot, Oxfordshire OX11 OQX, United Kingdom \\ ${ }^{5}$ Department of Physics, SUPA, University of Strathclyde, Glasgow G4 ONG, United Kingdom \\ ${ }^{6}$ Blackett Laboratory, Imperial College, London SW7 2AZ, United Kingdom \\ ${ }^{7}$ Dipartimento SBAI, Università di Roma "La Sapienza," 00161 Rome, Italy \\ ${ }^{8}$ Helmholtz. Institut Jena, D-07743 Jena, Germany \\ ${ }^{9}$ Institute of Physics of the ASCR, ELI-Beamlines Project, Na Slovance 2, 18221 Prague, Czech Republic
}

(Received 17 August 2013; accepted 4 February 2014; published online 11 March 2014)

\begin{abstract}
A modification to the standard Thomson parabola spectrometer is discussed, which is designed to measure high energy (tens of $\mathrm{MeV} /$ nucleon), broad bandwidth spectra of multi-species ions accelerated by intense laser plasma interactions. It is proposed to implement a pair of extended, trapezoidal shaped electric plates, which will not only resolve ion traces at high energies, but will also retain the lower energy part of the spectrum. While a longer (along the axis of the undeflected ion beam direction) electric plate design provides effective charge state separation at the high energy end of the spectrum, the proposed new trapezoidal shape will enable the low energy ions to reach the detector, which would have been clipped or blocked by simply extending the rectangular plates to enhance the electrostatic deflection. ( 2014 AIP Publishing LLC. [http://dx.doi.org/10.1063/1.4866021]
\end{abstract}

\section{INTRODUCTION}

High power lasers are nowadays able to accelerate protons and heavier ions up to tens of $\mathrm{MeV}$ per nucleon via various mechanisms such as target normal sheath acceleration (TNSA), ${ }^{1}$ radiation pressure acceleration (RPA) ${ }^{2}$ and break-out afterburner acceleration (BOA). ${ }^{3}$ Typically these mechanisms accelerate simultaneously several ion species, originated not only from the bulk target material, but also from the contaminant layers present on both sides of the target foils. Therefore, such ion sources are typically multi-species (protons, several charge states of carbon, oxygen, and the bulk target elements) and demand spectrometers with adequate charged species discrimination capability. Diagnosing and characterising spectra of individual ion species is key to understanding the underlying acceleration mechanisms, such as TNSA, RPA, BOA, etc., which are currently the object of extensive experimental investigations. Among ion diagnostics routinely used in such experiments, such as Radiochromic film stack detectors, ${ }^{4}$ nuclear activation techniques ${ }^{5}$ and Faraday cups, ${ }^{6}$ Thomson Parabola Spectrometers (TPS) are particularly useful as different ion species are dispersed in energy and mass-to-charge $(\mathrm{A} / \mathrm{Z})$ ratio by means of static electric and magnetic fields. ${ }^{7-9}$ Implementation of the diagnostic and data analysis are also relatively simple and straightforward.

The dispersion of ions in a TPS is governed by the electric and magnetic field strengths as well as the dimensions of

a)Electronic mail: s.kar@qub.ac.uk various elements, such as the field regions, and the distances from the electric and magnetic fields to the detector. Compact (in order to fit inside the vacuum interaction chamber) spectrometers are unable to provide sufficient $\mathrm{A} / \mathrm{Z}$ resolution for heavy ions beyond several $\mathrm{MeV} /$ nucleon (as the parabolic ion traces start to overlap), even when the energy dispersion by the magnetic field is adequate. Species separation can be increased primarily by increasing either the electric field strength or the longitudinal extent of the electric field region. However, the large electric field required for separating high energy ion tracks will cause lower energy ions to be deflected away from the detector, or to be clipped by the electric plate itself. Hence, the possibility of obtaining a full energy spectrum is compromised. In this paper we discuss how shaping of the electric field plates can enable both low and high energy ions to be retained while providing significant species separation at the high energy end.

The paper is structured as follows. After a brief discussion of the basic principle of a TPS diagnostic, Sec. III describes the particle tracing simulation employed to study different electric plate geometries. Section IV describes various methods of increasing the species separation and the derived trapezoidal plate design.

\section{WORKING PRINCIPLES}

A schematic of a traditional TPS used in laser plasma experiments is shown in Fig. 1. It consists of pairs of permanent rectangular magnets and electrodes, producing uniform magnetic and electric fields dispersing ions according to 


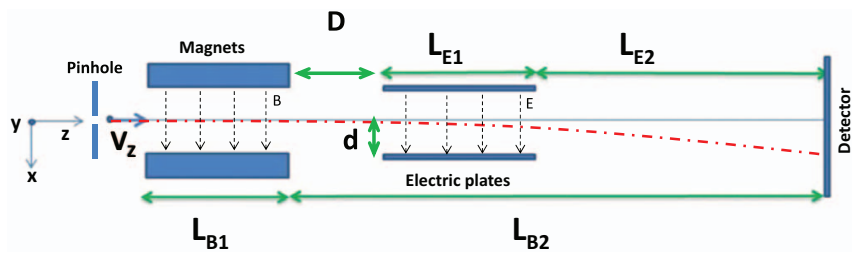

FIG. 1. Schematic of a Thomson parabola spectrometer (TPS).

their mass-to-charge ratio and energy, ${ }^{7}$ resulting in a unique parabolic trace on the detector plane for each mass-to-charge ratio. The magnetic and electric fields are parallel to each other, but perpendicular to the ion's initial momentum. The pinhole selects a pencil beam of ions as input to the TPS.

For uniform electric and magnetic fields, without considering fringe field effects, the ion displacements at the detector plane along the $\mathrm{Y}$ (owing to magnetic field) and $\mathrm{X}$ (owing to electric field) axes can be derived, respectively, for the nonrelativistic case as

$$
\begin{gathered}
S_{B}=\alpha B\left(\frac{L_{B 1}^{2}}{2}+L_{B 1} L_{B 2}\right), \\
S_{E}=\alpha^{2} E \frac{m}{q}\left(\frac{L_{E 1}^{2}}{2}+L_{E 1} L_{E 2}\right),
\end{gathered}
$$

where $\alpha=q / m v_{z}$ and $v_{z}$ is the longitudinal speed of the ion (of mass $m$ and charge $q$ ) at the pinhole. $L_{B 1}, L_{B 2}, L_{E 1}$, and $L_{E 2}$ correspond to the distances shown in Fig. 1, and $E$ and $B$ denote the electric and magnetic field strengths, respectively. The displacements owing to the magnetic $\left(S_{B}\right)$ and electric fields $\left(S_{E}\right)$ are derived using equations of motion for the particles and the Lorentz force, where one can neglect the change in $v_{z}$ without any loss of generality. Equations (1) and (2) show that the resulting displacements have a linear dependence on the magnetic $(B)$ and electric $(E)$ field strengths, whereas they have a stronger dependence on the length of either the magnets or the electric plates. In this paper we have focused on the electric field displacement, considering that the energy dispersion produced by the magnetic field is adequate. For instance, in order to benchmark the results discussed in this paper, we have considered a traditional TPS ${ }^{7,8}$ design with a pair of $50 \mathrm{~mm}$ square magnets providing a peak magnetic field of $1.0 \mathrm{~T}$, and $L_{B 2}=310 \mathrm{~mm}$. The magnetic dispersion of this system is shown in Fig. 2.

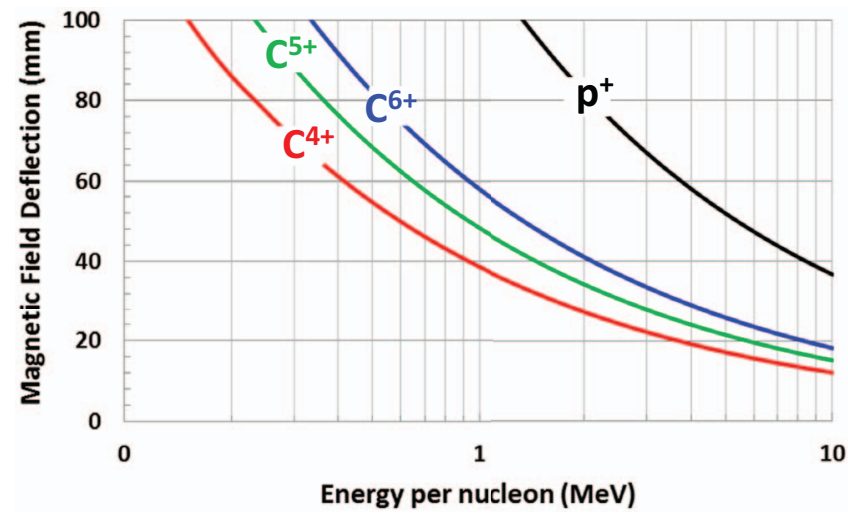

FIG. 2. Displacement of ions produced in the TPS by a pair of $50 \times 50 \mathrm{~mm}$ magnets of $1.0 \mathrm{~T}$ field strength at a distance of $L_{B 2}=310 \mathrm{~mm}$.
As mentioned earlier, in the case of a multi-species ion source, electric field dispersion is equally important for resolving ion spectra. If the deflection owing to the electric field is insufficient, it will be difficult to discriminate individual ion species due to overlapping of the parabolic traces towards the higher energy end. In order to study a suitable field configuration for effective charge state separation, while retaining low energy ions, we used the 3D particle tracing code PTRACE, ${ }^{10}$ as discussed below.

\section{SIMULATION DESIGN}

The particle tracing code PTRACE, ${ }^{10}$ written in $\mathrm{C}++$ object oriented methodology, computes the trajectory of a particle through a customised 3D electromagnetic field region using a differential equation solver at its core. The numerical algorithm is a fourth order Runge-Kutta solver coupled with an adaptive step-size monitoring routine. Such an approach offers a fast and reliable solution to the equation of motion, with the possibility of prescribing the precision to which the trajectory is computed. ${ }^{10}$

The 3D setup of the TPS was modeled in PTRACE as per the schematic shown in Fig. 1. The pencil beam of ions, selected by the pinhole of the TPS, was defined in PTRACE as a point ion source with multi-energy spectrum and very low divergence. The magnetic field object was defined next to the source. In order to include fringe fields, the magnetic field in this region was defined according to the field profile in a conventional magnet-yoke configuration, as measured by Carroll et al. ${ }^{11}$ In this case the magnetic field profile along the ion propagation axis ( $\mathrm{Z}$-axis) can be fitted with a super-Gaussian like function:

$$
B(z)=B_{\text {peak }} \exp \left[-\left(\frac{z-z_{\text {centre }}}{B_{F W H M} / 1.82}\right)^{4}\right],
$$

where $B_{\text {peak }}$ is the peak magnetic field, $z_{\text {centre }}$ is the $z$ coordinate of the center of the magnets, and $B_{F W H M}$ is the full width at half maximum of the field profile, which is approximately equal to the length of the magnets.

In order to study the effect of different charged plate geometries, a simple numerical approach for electric field calculation was implemented in the electric field object. As shown in Fig. 3, the electric field at any given point in space $P(x, y, z)$ is numerically calculated by superposition of the

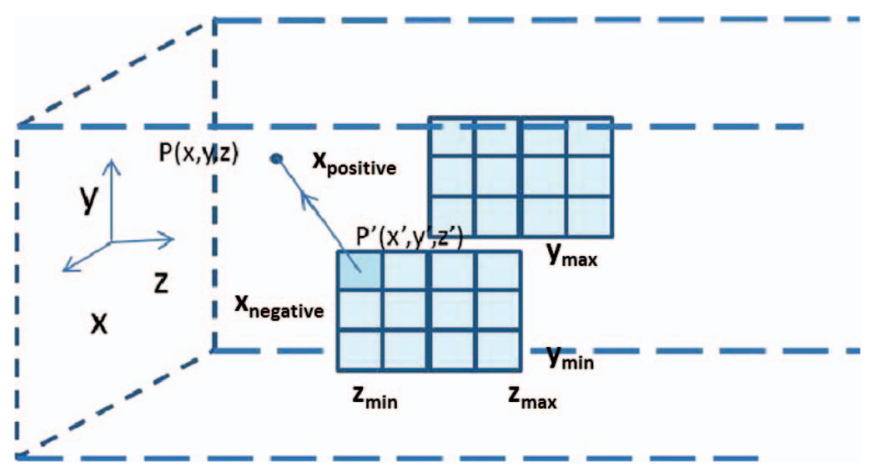

FIG. 3. Schematic of the electric plate setup in PTRACE for calculation of the E-field. 
electric fields due to all points on the charged plates. For example, in the case of a rectangular geometry, the electric field $\vec{E}$ due to the negative electrode will therefore be

$$
\vec{E}=\left.K \sigma \int_{y_{\min }}^{y_{\max }} \int_{z_{\min }}^{z_{\max }} \frac{\mathrm{d} y^{\prime} \mathrm{d} z^{\prime} \overrightarrow{P P^{\prime}}}{\left|\overrightarrow{P P^{\prime}}\right|^{3}}\right|_{x=x_{\text {negative }}},
$$

where $K=1 / 4 \pi \epsilon_{0}$, the surface charge density $\sigma=2 \epsilon_{0} E$ and the limits $y_{\max }, y_{\min }, z_{\max }, z_{\min }$, and $x_{\text {negative }}$ refer to those shown in Fig. 3. In order to simplify the calculation, the integration over $z$ was substituted with a summation along $z$. During the transit of an ion of a given mass, charge, and energy, the main module of PTRACE accesses the electric and magnetic field objects in order to obtain the fields at the ion's position. The trajectory of the ion is subsequently simulated by the differential equation solver. The detector plane was defined at a given distance from the electric plates, and PTRACE produced a $2 \mathrm{D}$ image of the simulated ion traces at this position.

\section{INCREASING SPECIES SEPARATION}

In principle, the species separation can be increased by three possible methods: (1) increasing the distance $L_{E 2}$, (2) increasing the electric field strength, and (3) increasing $L_{E 1}$. In terms of the capability of discriminating ion traces at the detector plane, one can define the resolution of the spectrometer as

$$
R=\left|\frac{S_{E 2}-S_{E 1}}{a}\right|=\frac{E}{2 a}\left|\frac{m_{2}}{q_{2}}-\frac{m_{1}}{q_{1}}\right| \alpha^{2}\left(L_{E 1}+2 L_{E 2}\right) L_{E 1},
$$

where $S_{E 1}$ and $S_{E 2}$ stand for the electric field displacements of two ion species of charges $q_{1}$ and $q_{2}$, and masses $m_{1}$ and $m_{2}$, respectively, at a given magnetic displacement (i.e., when $\left.q_{1} / m_{1} v_{1 z}=q_{2} / m_{2} v_{2 z}\right) . a$ stands for the size of the pinhole at the entrance of the spectrometer, which typically defines the width of the ion tracks at the detector (in a standard configuration, the pinhole to detector distance is significantly less than the distance between the ion source and the pinhole). Since $R=1$ corresponds to the threshold for discriminating two neighbouring ion tracks, one can simply rewrite Eq. (5) as

$$
\Delta\left(\frac{A}{Z}\right)=\frac{4 a[\mu \mathrm{m}]\left(A_{i} / Z_{i}\right)^{2} W_{i}[\mathrm{MeV} / \text { nucleon }]}{E[\mathrm{MV} / \mathrm{m}]\left(L_{E 1}[\mathrm{~mm}]+2 L_{E 2}[\mathrm{~mm}]\right) L_{E 1}[\mathrm{~mm}]},
$$

where $A_{i}, Z_{i}$, and $W_{i}$ represent the atomic mass, ionisation state, and energy per nucleon of a given charge species. As can be seen from Eq. (5), the resolution increases linearly with $E$ and $L_{E 2}$, whereas it has a quadratic dependency with the electric field plate length $\left(L_{E 1}\right)$. Increasing $L_{E 2}$ by a significant amount, in order to improve the resolution, is normally not a viable option for a number of reasons. For example, it could be practically difficult to field a spectrometer with a large $L_{E 2}$ inside a small interaction chamber, while keeping the TPS at a reasonable distance from the interaction target. Nevertheless, moving the detector plane significantly further away from the end of the electric field plates will increase the magnetic field

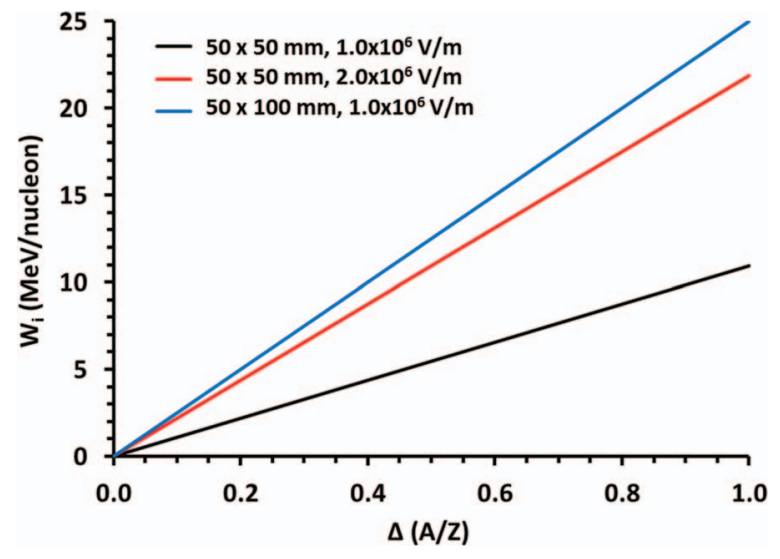

FIG. 4. Comparison of the maximum energy of $\mathrm{A} / \mathrm{Z}=2.0$ (for instance, $\mathrm{C}^{6+}$ ) resolvable for different values of $\Delta(A / Z)$ achieved with $50 \times 50 \mathrm{~mm}$ plates at $1 \times 10^{6} \mathrm{~V} / \mathrm{m}, 50 \times 50 \mathrm{~mm}$ plates at $2 \times 10^{6} \mathrm{~V} / \mathrm{m}$, and $50 \times 100$ $\mathrm{mm}$ plates at $1 \times 10^{6} \mathrm{~V} / \mathrm{m}$, assuming $L_{E 2}=150 \mathrm{~mm}$.

dispersion commensurately, as it will entail an increase in $L_{B 2}$, which may not be desired given a finite dimension of the detector. At the same time, as with any alteration that would increase the dispersion of the ion traces, there will be a decrease in the particle flux at the detector plane giving rise to a lower signal to noise ratio.

The electric field strength can be increased either by decreasing the separation of the electrodes or by increasing the voltage to the electrodes. However, the limiting factor is the onset of arcing/discharge between the electrodes, which can cause an instantaneous drop or fluctuations in the electric field. From experience, up to $\sim 2 \times 10^{6} \mathrm{~V} / \mathrm{m}$ can be applied in a typical experimental chamber at a vacuum pressure of $10^{-4}$ to $10^{-5}$ mbar without any significant discharge between the electrodes. ${ }^{9}$ On the other hand, increasing the length of the electric plates is not limited by any fundamental constraints.

The maximum energy, $W_{i}$, at which a particular charge state of ion species is resolvable from adjacent traces, can be calculated from Eq. (6). This is illustrated by Fig 4, where $W_{i}$ for $A / Z=2.0\left(\mathrm{C}^{6+}\right.$ for instance $)$ is plotted against $\Delta(A / Z)$ for different TPS configurations. For example, if we consider the neighbouring ion tracks of $\mathrm{C}^{6+}(A / Z=2.0)$ and $\mathrm{O}^{7+}$ $(A / Z=2.29)$, corresponding to $\Delta(A / Z)=0.29$, using $100 \mathrm{~mm}$ electric plates will provide track separation at significantly higher ion energies compared to using $50 \mathrm{~mm}$ long electric plates with the same electric field strength.

In order to illustrate the effect of increasing electric plate length on charge state separation, the performance of a TPS was simulated with PTRACE by changing the electric plate length, while keeping $B_{\text {peak }}, L_{B 1}$, and $L_{B 2}$ constant. One can see the significant improvement in separation between the traces at the high energy end (for example, around $100 \mathrm{MeV}$ protons and $200 \mathrm{MeV} \mathrm{C}^{6+}$ ions) for $150 \mathrm{~mm}$ long electric plates as shown in Fig. 5(b) as compared to the output of the standard TPS configuration shown in Fig. 5(a). However, it must be noted that the simulations assumed a point source entering the TPS, when in reality the pinhole will determine a finite track size at the detector and thus the highest energies which can be resolved by the TPS will generally be lower than those predicted by the simulation. 


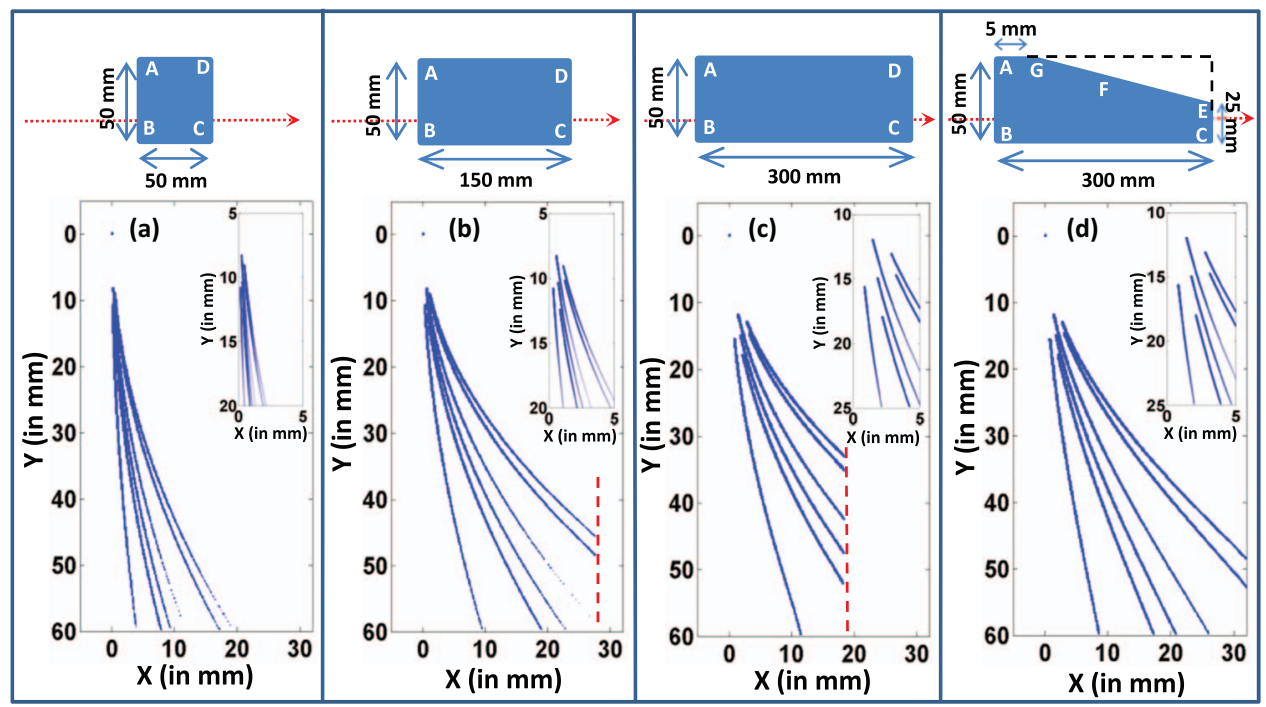

FIG. 5. Comparison between the Thomson parabola traces for several ion species (proton, $\mathrm{C}^{6+}, \mathrm{C}^{5+}, \mathrm{C}^{4+}, \mathrm{Au}^{40+}$, and $\mathrm{Au}{ }^{45+}$ from left to right, with energy up to $100 \mathrm{MeV}, 200 \mathrm{MeV}$, and $1000 \mathrm{MeV}$ for protons, carbon ions, and gold ions, respectively) obtained using different electric plate designs as shown in schematics above the simulated images: (a) rectangular plates with $L_{E 1}=50 \mathrm{~mm}$ and $L_{E 2}=250 \mathrm{~mm}$, (b) rectangular plates with $L_{E 1}=150 \mathrm{~mm}$ and $L_{E 2}=150 \mathrm{~mm}$, (c) rectangular plates with $L_{E 1}=300 \mathrm{~mm}$ and $L_{E 2}=150 \mathrm{~mm}$, and (d) trapezoidal plates with $L_{E 1}=300 \mathrm{~mm}$ and $L_{E 2}=150 \mathrm{~mm}$. The red arrows in the schematics of the electric plate design show the ion beam direction, with the beam axis $10 \mathrm{~mm}$ above the bottom edge of the electric plates. Other parameters used in these simulations are $L_{B 1}=50 \mathrm{~mm}, B=1.0 \mathrm{~T}, D=5 \mathrm{~mm}, E=1.8 \times 10^{6} \mathrm{~V} / \mathrm{m}$, and $d=9 \mathrm{~mm}$. The insets in the simulated images show the zoomed view of the high energy portions of the ion tracks. One can see the improvement in track separation in (b) as compared to (a), which is achieved by using a longer electric field region at the same electric field strength. Some clipping is here observed in the low energy part of the gold ion tracks, as shown by the dotted line in (b). The track separation at higher ion energies as well as the A/Z resolution of the system can be increased further by using even longer electric plates, as shown in (c), which may be required in future experiments with upcoming facilities. However, clipping of the low energy part of the ion tracks will become more serious in this case, as shown by the dotted red line. Trapezoidally shaped electric plates, as shown in (d), can be used in such high resolution spectrometers allowing the lower energy ions to pass over the top of the plate, while keeping the same track separation for the high energy ions obtained in (c).

In order to benchmark experimentally the performance of longer electric plates towards charge state separation, the TPS with $150 \mathrm{~mm}$ long electric plates (as shown in Fig. 6(a)) and a Fuji BAS-TR image plate detector were employed in
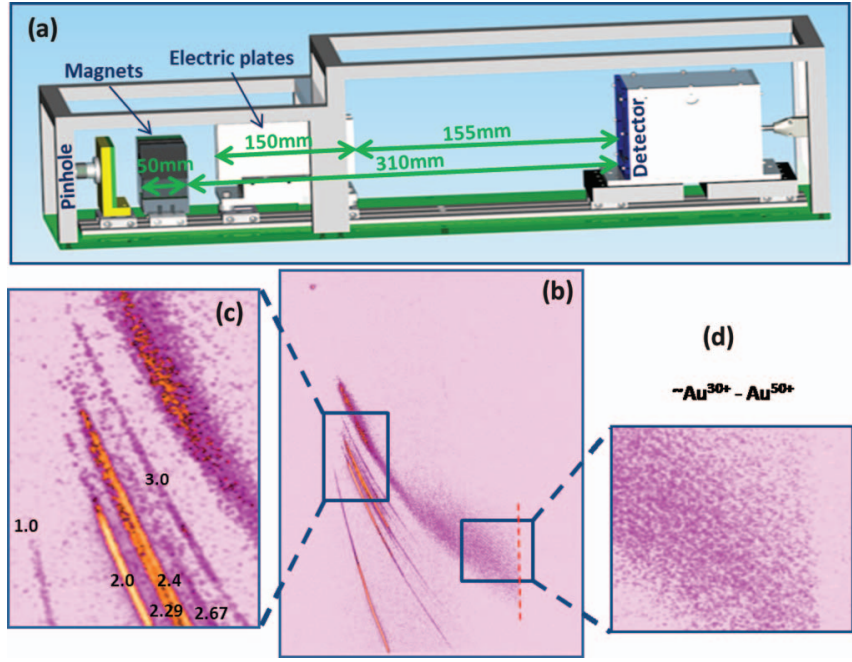

FIG. 6. (a) 3D schematic view of the compact TPS fielded in the experiment; (b) data obtained by the TPS showing traces of different energy and mass-tocharge ratio ions produced by the interaction of the Vulcan laser at intensity $3 \times 10^{20} \mathrm{~W} / \mathrm{cm}^{2}$ with a $10 \mathrm{~nm}$ thick Au foil. The low energy ions are clipped by the electric plate as shown by the dotted line; (c) a zoomed in view of the enhanced species separation produced by the $150 \mathrm{~mm}$ rectangular electric plates, where the numbers represent $A / Z$ values for the ion species, such as 1.0 - protons; $2.0-\mathrm{C}^{6+}$ and $\mathrm{O}^{8+} ; 2.29-\mathrm{O}^{7+} ; 2.4-\mathrm{C}^{5+} ; 2.67-\mathrm{O}^{6+} ; 3.0-$ $\mathrm{C}^{4+}$; (d) a zoomed in view of the fine charge state separation of $\mathrm{Au}$ ions, which are clipped by the longer electric plate. a recent experiment at the Vulcan Petawatt laser facility of RAL-STFC, UK. The laser delivered $\sim 250 \mathrm{~J}$ of energy on target in a 0.75 ps pulse duration. The laser was focused on to the target by a $f / 3$ off axis parabola, irradiating normally $10 \mathrm{~nm}$ thick Au foils at an intensity of $3 \times 10^{20} \mathrm{~W} / \mathrm{cm}^{2}$. The TPS was placed at a distance of $1.7 \mathrm{~m}$ from the laser irradiated target with a $100 \mu \mathrm{m}$ pinhole at the front. The applied electric and magnetic fields in this case were $1.8 \times 10^{6} \mathrm{~V} / \mathrm{m}$ and $\sim 1.0 \mathrm{~T}$, respectively. As shown in the experimental data in Fig. 6(b), the TPS was able to resolve clearly, in addition to the protons and several charge states of carbon and oxygen ions (see Fig. 6(c)), tracks of Au ions with adjacent charge states ranging from $\sim 30$ to $\sim 50$ (see Fig. 6(d)).

It is to be noted that the dimensions and field strengths of the TPS were chosen in order to resolve the $\mathrm{C}^{6+}$ tracks at the maximum energies produced in the experiment, which is around $15-20 \mathrm{MeV} /$ nucleon, while working within the space constraints of the interaction chamber. This is a significant improvement in track separation at the high energy end of the spectrum compared to that obtained using a standard TPS, as shown in Figs. 5(a) and 5(b). Moreover, one can see that the highest energy at which $C^{5+}(A / Z \approx 2.4)$ is still separated from its adjacent $\mathrm{O}^{7+}(A / Z \approx 2.29)$ track is $\approx 6 \mathrm{MeV} /$ nucleon, which agrees well with the formula for $\Delta(A / Z)$ given in Eq. (6). Similarly, the energy at which $\mathrm{Au}^{40+}$ is still separated from the next charge state of $\mathrm{Au}$ (i.e., $\mathrm{Au}^{41+}$ ), corresponding to $\Delta(A / Z)=0.12$, is $\approx 1.5 \mathrm{MeV} /$ nucleon.

Due to the strong electric field deflection required for charged species separation at the high energy end of the spectrum, the low energy ions suffer a large deflection within 
the electric plates and get clipped at the output end of the electric plates. The clipping of low energy ions can be clearly seen in the simulated (Fig. 5(b)) and experimental data (Fig. 6(b)). The clipping of the ion tracks becomes more serious (as shown in the Fig. 5(c)) if one extends the electric field region further in order to achieve either track separation at higher energies or, to resolve ion tracks with smaller $\Delta(A / Z)$ values. Next generation facilities are expected to result in a significant increase of ion energies which will then demand such higher resolution spectrometers.

Using a longer electric field region instead of a higher electric field, in order to improve the resolution of the spectrometer, provides flexibility in terms of controlling the deflection for different energy ions. By reshaping the geometry of the electric plates, clipping of the lower energy ions can be avoided. Since the magnetic deflection, prior to the electric plates, disperses the ions according to their energy along the vertical direction ( $\mathrm{Y}$ axis), reshaping the electric plates into a trapezoidal geometry (for instance as shown in Fig. 5(d)) will allow the low energy ions to reach the detector, while the high energy ions will still experience the full length of the electric plates. In this way a full ion spectrum can be captured while obtaining the desired species separation at the high energy end of the spectrum, as shown in Fig. 5(d). Since different energy ions experience different lengths of electric plates in a trapezoidal geometry, the ion traces on the detector will not follow a parabolic shape. However, as long as the ion traces are well separated and their charge states can be identified, the spectral profile can be obtained by deconvolving the traces after binning the signal along the $\mathrm{Y}$ axis, i.e., along the axis of the energy dispersion caused by the magnetic field.

The dimensions of the trapezoidal electric plates can be chosen according to the energy dispersion of the TPS (which depends on $B, L_{B 1}$, and $L_{B 2}$ ) and the desired species separation at the detector plane. For example, the trapezoidal electric plates used in Fig. 5(d) were designed by modifying the rectangular electric plate of $300 \mathrm{~mm}$ shown in Fig. 5(c), assuming that this provides the desired species separation. Clipping of ions on the electric plate occurs when the displacement due to the electric field is equal to, or greater than, the distance between the beam axis and the negative plate, $d$, shown in Fig. 1. By using $L_{E 2}=0$ in Eq. (2), one can estimate the maximum energy $\left(W_{\max }\right)$ of a given ion species that would clip the negative plate. The magnetic field displacement for this energy at the end of the electric plates can be calculated by using $L_{B 2}=D+L_{E 1}$ in Eq. (1), where $D$ is the distance between the magnets and the electric plates as shown in Fig. 1. This defines the corner point "E" of the trapezoidal geometry shown in the schematic in Fig. 5(d). The point "G" is set according to the minimum energy $\left(W_{\min }\right)$ of the ion species detected by the detector. Using $L_{E 2}=0$ in Eq. (2), one can estimate $L_{E 1}$ (say $L_{E 1}^{\prime}$ ) for which $S_{E}=d$. The magnetic displacement (say $S_{B}^{\prime}$ ) of these ions at $L_{B 2}=D+L_{E 1}^{\prime}$, which can be calculated by using Eq. (1), will define the point " $F$ " on the negative plate where the ions of energy $W_{\min }$ will be clipped. The line EG can then be defined such that the edge of the electric plate is below the point "F" (i.e., below $S_{B}^{\prime}$ at $L_{B 2}=D+L_{E 1}^{\prime}$ ), thus allowing the lower energy ions to pass over the edge of the electric plate.
The manufacture of this type of electric plates is relatively cheap and easy as it merely requires cutting the corner off a standard rectangular plate. Once the cut has been made, the plate will have a fixed geometry which may be considered a disadvantage as the geometry was chosen with specific magnetic and electric field strengths in mind. However, the plates can be used for different magnetic and electric filed strengths while retaining the entire spectrum. For example, weaker electric fields and/or stronger magnetic fields can be accommodated by raising the plates up with respect to the beam axis, whereas, stronger electric fields and/or weaker magnetic fields can be accommodated by lowering the plates down with respect to the beam axis. Alternatively, different sets of plates could be manufactured for different field strengths, with shallower cuts made for strong electric and weak magnetic fields, and steeper cuts for weak electric and strong magnetic fields.

\section{CONCLUSION}

With the growing need for a compact, high resolution ion spectrometer for multi-species, multi- $\mathrm{MeV} /$ nucleon ion source, produced by high intensity laser plasma interactions, we have studied a potential modification to the standard Thomson parabola spectrometer currently used in experiments. Extended, trapezoidal shaped electric plates are proposed in contrast to conventional rectangular electric plates, which will provide not only better trace separation for high energy ions (due to the extended length of the electric plates), but will also retain the lower energy part of the spectrum. A compact TPS using long electric plates, benchmarked in a recent experiment, was capable of resolving different charge states of high energy gold ions produced by the interaction of a high power laser with ultra-thin gold foils. The data, as well as simulations of TPS design required by upcoming ion beam parameters, show clipping of low energy ions by the electric plates, which can be mitigated by using trapezoidal shaped electric plates.

\section{ACKNOWLEDGMENTS}

The authors acknowledge funding from EPSRC [EP/J002550/1-Career Acceleration Fellowship held by S.K., EP/L002221/1, EP/E035728/1, EP/K022415/1, EP/J003832/1, and EP/J500094/1], Laserlab Europe (EC-GA 284464), projects ELI (Grant No. CZ.1.05/1.1.00/483/02. 0061) and OPVK 3 (Grant No. CZ.1.07/2.3.00/20.0279). Authors also acknowledge the support of the mechanical engineering staff of the Central Laser Facility, STFC, UK.

\footnotetext{
${ }^{1}$ S. C. Wilks et al., Phys. Plasmas 8, 542 (2001); A. Macchi, M. Borghesi, and M. Passoni, Rev. Mod. Phys. 85, 751 (2013); J. Fuchs et al., Nat. Phys. 2, 48 (2006); L. Robson et al., ibid. 3, 58 (2007); M. Passoni, L. Bertagna, and A. Zani, New J. Phys. 12, 045012 (2010).

${ }^{2}$ T. Esirkepov, M. Borghesi, S. V. Bulanov, G. Mourou, and T. Tajima, Phys. Rev. Lett. 92, 175003 (2004); S. Kar et al., ibid. 100, 225004 (2008); A. P. L. Robinson, P. Gibbon, M. Zepf, S. Kar, R. G. Evans, and C. Bellei, New J. Phys. 10, 013021 (2008); A. P. L. Robinson, M. Zepf, S. Kar, R. G. Evans, and C. Bellei, Plasma Phys. Control. Fusion 51, 024004 (2009); A. Macchi, S. Veghini, T. V. Liseykina, and F. Pegoraro, New J. Phys. 12, 045013 (2010); B. Qiao, S. Kar, M. Geissler, P. Gibbon, M. Zepf, and M.
} 
Borghesi, Phys. Rev. Lett. 108, 115002 (2012); S. Kar et al., ibid. 109, 185006 (2012).

${ }^{3}$ L. Yin, B. J. Albright, B. M. Hegelich, and J. C. Fernández, Laser Part. Beams 24, 291-298 (2006); L. Yin, B. J. Albright, K. J. Bowers, D. Jung, J. C. Fernández, and B. M. Hegelich, Phys. Rev. Lett. 107, 045003 (2011); D. Jung et al., ibid. 107, 115002 (2011); B. Hegelich, "Towards GeV laserdriven ion acceleration," paper presented in Coulomb 2009, Senigallia, Italy, 15-19 June 2009.

${ }^{4}$ D. S. Hey, M. H. Key, A. J. Mackinnon, A. G. MacPhee, P. K. Patel, R. R. Freeman, L. D. Van Woerkom, and C. M. Casteneda, Rev. Sci. Instrum. 79, 053501 (2008); F. Nürnberg et al., ibid. 80, 033301 (2009).

${ }^{5}$ R. J. Clarke et al., Nucl. Instrum. Methods Phys. Res. A 585, 117-120 (2008).
${ }^{6}$ A. Kashefian Naieni, F. Bahrami, N. Yasrebi, and B. Rashidian, Vacuum 83, 1095-1099 (2009).

${ }^{7}$ M. Rhee, R. F. Schneider, and D. J. Weidman, Rev. Sci. Instrum. 58, 240 (1987); S. Sakabe, T. Mochizuki, T. Yamanaka, and C. Yamanaka, ibid. 51 , $1314(1980)$

${ }^{8}$ D. C. Carroll, P. Brummitt, D. Neely, F. Lindau, O. Lundh, C.-G. Wahlström, and P. McKenna, Nucl. Instrum. Methods Phys. Res. A 620, 23 (2010); K. Harres et al., Rev. Sci. Instrum. 79, 093306 (2008); J. Morrison, C. Willis, R. R. Freeman, and L. D. Van Woerkom, ibid. 82, 033506 (2011).

${ }^{9}$ D. Jung et al., Rev. Sci. Instrum. 82, 013306 (2011).

${ }^{10}$ A. Schiavi, Ph.D. thesis, Imperial College London, 2003.

${ }^{11}$ D. C. Carroll et al., CLF Annual report, 2005-2006, p. 16. 